\title{
Chronic typhoid infection and the risk of biliary tract cancer and stones in Shanghai, China
}

\author{
Mahboobeh Safaeian ${ }^{*}$, Yu-Tang Gao ${ }^{2}$, Lori C Sakoda ${ }^{3}$, Sabah M Quraishi ${ }^{1}$, Asif Rashid ${ }^{4}$, Bing-Shen Wang ${ }^{5}$, \\ Jinbo Chen ${ }^{6}$, James Pruckler ${ }^{7}$, Eric Mintz ${ }^{7}$ and Ann W Hsing ${ }^{1}$
}

\begin{abstract}
Previous studies have shown a positive association between chronic typhoid carriage and biliary cancers. We compared serum Salmonella enterica serovar Typhi antibody titers between biliary tract cancer cases, biliary stone cases without evidence of cancer, and healthy subjects in a large population-based case-control study in Shanghai, China.

Participants included 627 newly diagnosed primary biliary tract cancer patients; 1,037 biliary stone cases (774 gallbladder and 263 bile-duct) and 959 healthy subjects without a history of cancer, randomly selected from the Shanghai Resident Registry.

Overall only 6/2,293 (0.26\%) were Typhi positive. The prevalence of Typhi was 1/457 (0.22\%), 4/977 (0.41\%), and 1/ $859(0.12 \%)$ among cancer cases, biliary-stone cases, and population controls, respectively.

We did not find an association between Typhi and biliary cancer in Shanghai, due to the very low prevalence of chronic carriers in this population.

The low seroprevalence of S. Typhi in Shanghai is unlikely to explain the high incidence of biliary cancers in this population.
\end{abstract}

Biliary tract cancers, encompassing tumors of the gallbladder, extrahepatic bile ducts, and ampulla of Vater, are rare but highly fatal. Other than an association with a history of gallstones, the etiology of biliary tract cancer is obscure [1]. Several studies in India, a high-risk population for gallbladder cancer, have reported an association between chronic carriage of typhoid and biliary tract cancer [2,3]. Typhoid fever is caused by Salmonella enterica serovar Typhi, which can result in chronic typhoidal cholesystitis. About $3-4 \%$ of patients with acute Typhi infection become chronic, asymptomatic carriers [4]. To examine the relationship between chronic typhoid infection and biliary tract cancer, we compared serum antibodies to the $\mathrm{Vi}$ antigen of serovar Typhi, using the indirect hemagglutination (IHA) assay [5] between biliary tract cancer cases, biliary stone cases without evidence of cancer, and healthy subjects randomly selected from the general population, in a large population-based case-control study in Shanghai, China.

\footnotetext{
* Correspondence: safaeianm@mail.nih.gov

${ }^{1}$ Division of Cancer Epidemiology and Genetics, National Cancer Institute, Bethesda, Maryland, USA

Full list of author information is available at the end of the article
}

Details of the study have been reported elsewhere [6]. Briefly, between 1997 and 2001, using a rapid reporting system established in 42 collaborating hospitals in Shanghai, we enrolled 627 patients newly diagnosed with primary biliary tract cancer (ICD9 156), 1,037 biliary stone cases ( 774 gallbladder and 263 bile-duct) frequency-matched to cancer cases on age (5-year intervals), gender, and hospital, and 959 healthy subjects without a history of cancer, randomly selected from the Shanghai Resident Registry and frequency-matched to cancer cases on age (5-year intervals) and gender. Ninety-five percent of the eligible cases and $82 \%$ of the eligible controls participated in the study. The study protocol was approved by the Institutional Review Boards of the U.S. National Cancer Institute and the Shanghai Cancer Institute. All subjects provided written informed consent.

Determination of antibody status to the Vi capsular polysaccharide antigen of Typhi was assessed at the Centers for Disease Control and Prevention, Atlanta, GA. Titers $\geq 160$ were defined as positive for chronic typhoid infection. For quality control, 30 samples were blindly retested; concordance of these samples was $100 \%$. 
We assessed the association of chronic typhoid infection with biliary tract cancer and stones by case-control status (i) overall, and (ii) by anatomic sub-site. Univariate and multivariate odds ratios and $95 \%$ confidence intervals were calculated, using maximum likelihood methods.

The characteristics of study subjects are shown in Table 1. Cancer cases (regardless of sub-site) were more likely to have gallstones compared with cancer-free controls. Table 2 shows the prevalence of chronic typhoid infection by subject status. Of the 2,293 subjects, only 6 $(0.26 \%)$ were positive at $\geq 160$ titer. Four additional participants $(0.17 \%)$ were marginally positive ( $\geq 80$ titer). The prevalence of Typhi was $1 / 457$ (0.22\%), 4/977 $(0.41 \%)$, and $1 / 859(0.12 \%)$ among cancer cases, biliarystone cases, and population controls, respectively.

The low prevalence of chronic Typhi carriers in Shanghai largely explains the lack of association between biliary cancer and chronic carriage of Typhi in our study, suggesting that the rise in biliary tract cancer in Shanghai is likely due to other environmental or infectious agents. Biliary tract cancer is ranked $15^{\text {th }}$ in cancer incidence in China, which is similar to India (ranked $14^{\text {th }}$ ) and Bangladesh (ranked $12^{\text {th }}$ ). While it is hard to estimate incidence of typhoid fever, China is classified as a medium typhoid fever incidence country, compared with India which is classified as a high typhoid fever incidence country [7]. Thus, the very low prevalence of chronic typhoid carriage observed is consistent with the lower prevalence of acute typhoid fever reported in China compared with India and Pakistan [8], where high prevalence of Typhi chronic carriage has been reported $(\sim 2 \%)$. Misclassification of Typhi in cases and controls, due to the lower assay performance in a population where typhoid infection is rare (thus with lower titers among infected), is possible, although such misclassification should be non-differential among cases and controls. While the IHA assay has been well validated in high-prevalence chronic carriage settings, such as India, in a study from Vietnam, an area with high prevalence of acute typhoid fever, very low rates of serologic positivity among the general population were found [9], suggesting that the $\mathrm{Vi}$-antibody test may be a less useful tool for detection of chronic carriage of Typhi in lowprevalence chronic carriage settings.

Our study is the largest population-based study of biliary tract cancer. With nearly complete case ascertainment and a high response rate, selection bias is minimal in the study. Misclassification of the outcome (cancer and stones) is also minimal, due to the stringent case confirmation.

In summary, due to the very low prevalence of chronic S. typhi carriers in this population, we were unable to evaluate chronic Typhi carriage and biliary cancer in Shanghai. In populations where chronic carriage of Typhi is rare, a much larger sample size and a more sensitive assay are needed to confirm the typhoid hypothesis. However, we are not able to rule out the possibility that chronic typhoid infection is associated with biliary tract cancer in populations with frequent outbreaks or a high prevalence of typhoid fever.

Table 1 Selected characteristics of cases and controls, Shanghai, China

\begin{tabular}{|c|c|c|c|c|c|c|c|c|c|c|c|c|}
\hline \multirow{3}{*}{ Characteristics } & & & \multicolumn{6}{|c|}{ Biliary Tract Cancer } & \multicolumn{4}{|c|}{ Biliary Stones } \\
\hline & \multicolumn{2}{|c|}{ Controls } & \multicolumn{2}{|c|}{ Gallbladder } & \multicolumn{2}{|c|}{ Extrahepatic Bile Duct } & \multicolumn{2}{|c|}{ Ampulla of Vater } & \multicolumn{2}{|c|}{ Gallbladder Stones } & \multicolumn{2}{|c|}{ Bile Duct Stones } \\
\hline & $\mathrm{N}$ & $\%$ & $\mathbf{N}$ & $\%$ & $\mathbf{N}$ & $\%$ & $\mathbf{N}$ & $\%$ & $\mathrm{~N}$ & $\%$ & $\mathbf{N}$ & $\%$ \\
\hline Total & 859 & 100.0 & 262 & 100.0 & 140 & 100.0 & 55 & 100.0 & 728 & 100.0 & 249 & 100.0 \\
\hline \multicolumn{13}{|l|}{ Age } \\
\hline$<55$ & 113 & 13.2 & 36 & 13.7 & 21 & 15.0 & 5 & 9.1 & 230 & $31.6^{*}$ & 56 & $22.5^{*}$ \\
\hline $55-64$ & 247 & 28.8 & 64 & 24.4 & 37 & 26.4 & 13 & 23.6 & 207 & 28.4 & 75 & 30.1 \\
\hline$\geq 65$ & 499 & 58.1 & 162 & 61.8 & 82 & 58.6 & 37 & 67.3 & 291 & 40.0 & 118 & 47.4 \\
\hline \multicolumn{13}{|l|}{ Sex } \\
\hline Male & 335 & 39.0 & 72 & $27.5^{*}$ & 84 & $60.0^{*}$ & 30 & $54.6^{*}$ & 250 & $34.3^{*}$ & 120 & 48.2 \\
\hline Female & 524 & 61.0 & 190 & 72.5 & 56 & 40.0 & 25 & 45.5 & 478 & 65.7 & 129 & 51.8 \\
\hline Education (\% > Middle school) & 293 & 34.1 & 57 & 21.8 & 45 & 32.4 & 16 & 29.1 & 313 & 43.0 & 94 & 37.8 \\
\hline Smoking $(\%)+$ & 259 & 30.2 & 69 & 26.4 & 63 & $45.0^{*}$ & 25 & $45.5^{*}$ & 177 & $24.3^{*}$ & 92 & 37.0 \\
\hline Alcohol Use (\%)‡ & 177 & 20.6 & 42 & 16.0 & 45 & $32.1^{*}$ & 14 & 25.5 & 110 & $15.1^{*}$ & 48 & 19.4 \\
\hline Diabetes (\%) & 71 & 8.3 & 34 & $13.0^{*}$ & 12 & 8.6 & 3 & 5.5 & 78 & $10.7^{*}$ & 29 & $11.7^{*}$ \\
\hline Hypertension (\%) & 360 & 41.9 & 94 & 35.9 & 41 & $29.3^{*}$ & 14 & $25.5^{*}$ & 249 & 34.2 & 70 & $28.1^{*}$ \\
\hline Gallstones (\%) & 150 & 17.5 & 222 & $84.7^{*}$ & 93 & $66.4^{*}$ & 29 & $52.7^{*}$ & - & - & - & - \\
\hline Body Mass Index $(\% \geq 25.0)$ & 251 & 29.3 & 104 & 39.9 & 32 & 22.9 & 19 & 34.6 & 271 & 37.3 & 90 & 36.3 \\
\hline
\end{tabular}

* Chi-sq test comparing cases and controls, $p<0.05$

† Current smokers, defined as those who were smoking cigarettes regularly at the time of interview

₹ Current drinkers, defined as those drinking alcohol regularly at the time of interview 
Table 2 Odds ratios* (ORs) and 95\% confidence intervals (CI) for cancers of the biliary tract cancer and stones in relation to chronic typhoid infection, Shanghai China

\begin{tabular}{|c|c|c|c|c|c|c|c|c|c|}
\hline \multirow[b]{3}{*}{ Typhoid } & \multicolumn{5}{|c|}{ Biliary Tract Cancer } & \multicolumn{4}{|c|}{ Biliary Stones } \\
\hline & Controls $^{a}$ & Total & Gallbladder & $\begin{array}{l}\text { Extrahepatic Bile } \\
\text { Duct }\end{array}$ & $\begin{array}{l}\text { Ampulla of } \\
\text { Vater }\end{array}$ & Controls $^{b}$ & $\begin{array}{l}\text { Biliary } \\
\text { Stones }\end{array}$ & $\begin{array}{l}\text { Gallbladder } \\
\text { Stones }\end{array}$ & $\begin{array}{l}\text { Bile Duct } \\
\text { Stones }\end{array}$ \\
\hline & $\mathbf{N}$ & N OR $(95 \% \mathrm{Cl})$ & $\begin{array}{l}\text { N OR } \\
(95 \% \mathrm{Cl})\end{array}$ & N OR $(95 \% C l)$ & $\begin{array}{l}\text { N OR } \\
(95 \% \mathrm{Cl})\end{array}$ & $\mathbf{N}$ & $\begin{array}{l}\text { N OR } \\
(95 \% \mathrm{Cl})\end{array}$ & N OR $(95 \% C l)$ & $\begin{array}{l}\text { N OR } \\
(95 \% \mathrm{Cl})\end{array}$ \\
\hline Total & 859 & 457 & 262 & 140 & 55 & 654 & 977 & 728 & 249 \\
\hline Negative $^{c}$ & 858 & 456 & 262 & 139 & 55 & 654 & 973 & 726 & 247 \\
\hline \multicolumn{10}{|l|}{ Positive } \\
\hline$\geq 1: 160^{d}$ & 1 & $11.9(0.1-31.1)$ & 0 & $15.9(0.4-95.2)$ & 0 & 0 & 4 & 2 & 2 \\
\hline
\end{tabular}

*OR = odds ratio, adjusted for age and for level of education

${ }^{\mathrm{a}} \mathrm{N}=800$ for gallbladder cancer controls due to exclusion of those with history of cholecystectomy

${ }^{\mathrm{b}}$ Controls with gallstones were excluded.

c Negative defined as no hemagglutination at $<160$.

d Positive defined as hemagglutination at $\geq 160$.

Given that gallstone is a common disease worldwide, affecting $15 \%$ of the population, and that over 17 million people worldwide are affected by typhoid fever, the role of Typhi infection in the development of gallstones and biliary tract cancer needs further clarification.

\section{Acknowledgements}

We thank Jie Deng, Jiarong Cheng, Lu Sun, Kai Wu, and the staff at the Shanghai Cancer Institute for data collection, specimen collection, and processing, surgeons at the collaborating hospitals for data collection, and local pathologists for pathology review; Shelley Niwa of Westat for data preparation and management; and Janis Koci of the Scientific Applications International Corporation for management of the biological samples.

This project has been funded in whole or in part with federal funds from the National Cancer Institute, National Institutes of Health, under contract N01-CO-12400. The content of this publication does not necessarily reflect the reviews or policies of the Department of Health and Human Services, nor does mention of trade names, commercial products, or organizations imply endorsement by the U.S. Government.

\section{Author details}

'Division of Cancer Epidemiology and Genetics, National Cancer Institute, Bethesda, Maryland, USA. ${ }^{2}$ Department of Epidemiology, Shanghai Cancer Institute, Shanghai Cancer Institute, Shanghai, China. ${ }^{3}$ Department of Epidemiology, University of Washington, Seattle, WA, USA. ${ }^{4}$ Department of Pathology, the University of Texas MD Anderson Cancer Center, Houston, TX USA. ${ }^{5}$ Department of Surgery, Zhongshan Hospital, Shanghai Medical University, Shanghai, China. ${ }^{6}$ Department of Epidemiology and Biostatistics, University of Pennsylvania, Philadelphia, PA, USA. ${ }^{7}$ Diarrheal Diseases Epidemiology Section, Foodborne and Diarrheal Diseases Branch, Centers for Disease Control and Prevention, Atlanta, GA, USA.

\section{Authors' contributions}

MS: responsible for analysis and manuscript preparation. YTG: PI of the study (China), responsible for study design, subject recruitment, conduct of the study and all aspects of the study in China. BSW: surgeon, responsible for patient recruitments, diagnosis of cancer, and treatment of cases. EM and JP: - responsible for development of assays, QC of assays, running of assays and accuracy of results. AR: study pathologist. Responsible for the diagnosis of all cancer cases, QC, and review of slides. LS and SQ - responsible for the conduct of the study, data management, specimen management and retrieval, implementation of QCs. JC: contributed to the statistical analysis and design of the study. $\mathrm{AH}$ - responsible for all aspects of the study, including study design, subject recruitment, conduct of the study, analysis, and preparation of the manuscript All authors contributed to data interpretation and read and approved the final manuscript.

\section{Competing interests}

The authors declare that they have no competing interests.

Received: 13 December 2010 Accepted: 2 May 2011

Published: 2 May 2011

\section{References}

1. Hsing AW, Rashid A, Devesa SS, Fraumeni JF Jr: Biliary tract cancer. In Cancer Epidemiology and Prevention. Edited by: Schottenfeld D, Fraumeni JF Jr. Oxford University Press; 2006:787-800.

2. Dutta U, Garg PK, Kumar R, Tandon RK: Typhoid carriers among patients with gallstones are at increased risk for carcinoma of the gallbladder. Am J Gastroenterol 2000, 95:784-787

3. Vaishnavi C, Kochhar R, Singh G, Kumar S, Singh S, Singh K: Epidemiology of typhoid carriers among blood donors and patients with biliary, gastrointestinal and other related diseases. Microbiol Immunol 2005 49:107-112.

4. Lin FY, Becke JM, Groves C, Lim BP, Israel E, Becker EF, Helfrich RM, Swetter DS, Cramton T, Robbins JB: Restaurant-associated outbreak of typhoid fever in Maryland: identification of carrier facilitated by measurement of serum Vi antibodies. J Clin Microbiol 1988, 26:1194-1197.

5. Lanata CF, Levine MM, Ristori C, Black RE, Jimenez L, Salcedo M, Garcia J, Sotomayor V: Vi serology in detection of chronic Salmonella typhi carriers in an endemic area. Lancet 1983, 2:441-443.

6. Gao YT, Han TQ, Rashid A, Sakoda LC, Wang BS, Shen MC, Zhang BH, Niwa S, Chen J, Fraumeni JF Jr: Gallstones and the risk of biliary tract cancer: a population-based study in China. Br J Cancer 2007, 97:1577-1582.

7. Crump JA, Luby SP, Mintz ED: The global burden of typhoid fever. Bull World Health Organ 2004, 82(5):346-53.

8. Acosta CJ, Danovaro-Holliday MC, Baiqing D, Bhattacharya SK, Agtini MD, Bhutta ZA, Canh DG, Ali M, Shin S, Wain J, Page AL, Albert MJ, Farrar J, buElyazeed R, Pang T, Galindo CM, vonSeidlein L, Clemens JD: A study of typhoid fever in five Asian countries: disease burden and implications for controls. Bull World Health Organ 2008, 86:260-268.

9. Gupta A, My Thanh NT, Olsen SJ, Sivapalasingam S, My Trinh TT, Phuong Lan NT, Hoekstra RM, Bibb W, Minh NT, Danh TP, Cam PD, Mintz ED: Evaluation of community-based serologic screening for identification of chronic Salmonella typhi carriers in Vietnam. Int J Infect Dis 2006, 10:309-314.

doi:10.1186/1750-9378-6-6

Cite this article as: Safaeian et al:: Chronic typhoid infection and the risk of biliary tract cancer and stones in Shanghai, China. Infectious Agents and Cancer 2011 6:6 